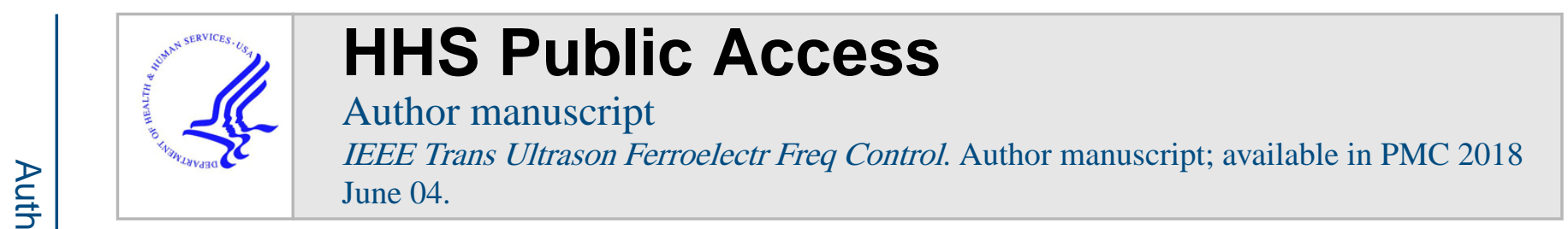

Published in final edited form as:

IEEE Trans Ultrason Ferroelectr Freq Control. 2017 January ; 64(1): 141-149. doi:10.1109/TUFFC. 2016.2640952.

\title{
Real-Time Intravascular Ultrasound and Photoacoustic Imaging
}

Donald VanderLaan ${ }^{*}$, Andrei Karpiouk ${ }^{*}$, Doug Yeager ${ }^{*}$, and Stanislav Emelianov ${ }^{\star}, \pm$ "Electrical \& Computer Engineering, Georgia Institute of Technology, Atlanta GA 30332, United States

\section{Abstract}

Combined intravascular ultrasound and photoacoustic imaging (IVUS/IVPA) is an emerging hybrid modality being explored as a means of improving the characterization of atherosclerotic plaque anatomical and compositional features. While initial demonstrations of the technique have been encouraging, they have been limited by catheter rotation and data acquisition, displaying and processing rates on the order of several seconds per frame as well as the use of off-line image processing. Herein, we present a complete IVUS/IVPA imaging system and method capable of real-time IVUS/IVPA imaging, with online data acquisition, image processing and display of both IVUS and IVPA images. The integrated IVUS/IVPA catheter is fully contained within a $1 \mathrm{~mm}$ outer diameter torque cable coupled on the proximal end to a custom-designed spindle enabling optical and electrical coupling to system hardware, including a nanosecond-pulsed laser with a controllable pulse repetition frequency capable of greater than $10 \mathrm{kHz}$, motor and servo drive, an ultrasound pulser/receiver, and a $200 \mathrm{MHz}$ digitizer. The system performance is characterized and demonstrated on a vessel-mimicking phantom with an embedded coronary stent intended to provide IVPA contrast within content of an IVUS image.

\section{Index Terms}

Intravascular ultrasound; photoacoustic; real-time; imaging system; atherosclerosis; coronary stent

\section{Introduction}

Coronary heart disease is responsible for greater than one in six deaths within the United States and remains among the leading causes of mortality throughout the industrialized world [1]. In many cases, the presence and extent of atherosclerotic plaques, the underlying culprit of coronary heart disease, goes undiagnosed prior to a patient suffering an acute coronary event such as a myocardial infarction [2]. This limitation is due, in part, to an inability of the most widely utilized clinical diagnostic techniques to adequately characterize coronary atheroma composition and effectively guide percutaneous coronary intervention of high-risk atherosclerotic lesions such as thin-capped fibroatheroma (TCFA). Over the past several decades, our understanding of the cellular and molecular mechanisms which promote coronary plaque progression and destabilization has improved significantly. Advances in

Personal use is permitted, but republication/redistribution requires IEEE permission. See http://www.iee.org/publications_standards/ publications/rights/index.html

${ }^{ \pm}$Corresponding author: stas@gatech.edu. 
molecular biology, combined with extensive clinical and preclinical research, have led to the evolution from a view of coronary atherosclerosis as sites of passive lipid accumulation and storage to a much more dynamic biological process. Plaque formation represents a long term progression involving endothelial dysfunction with low density lipoprotein infiltration, monocyte recruitment and subsequent foam cell formation, and over expression of destabilizing extracellular matrix proteins, all of which lead to the formation of a prothrombotic core which can induce a myocardial infarction and sudden cardiac death if exposed to the artery lumen [3-6].

Despite increased appreciation for the dynamic cellular and molecular processes involved in the development of a vulnerable atherosclerotic plaque, clinical diagnostic and interventional practices have failed to fully keep pace. Rather, the most common diagnostic imaging modalities, including contrast angiography, cardiac computed tomography, intravascular ultrasound (IVUS), and intravascular optical coherence tomography (OCT), provide clinicians with a view of vessel morphology, but each fails to provide an effective means of differentiating the underlying composition of an atherosclerotic plaque [7]. The limitations associated with basing diagnoses on the extent of arterial narrowing are made particularly evident by studies showing that arteries exhibit positive remodeling to maintain lumen area as plaques develop up to a total burden of $40 \%$ [8] and that extreme narrowing of arteries in the weeks or months before a myocardial infarction are found in only about $15 \%$ of cases [9]. IVUS imaging exhibits limited ultrasound contrast between lipid-rich tissues and benign ones and cannot reliably differentiate them [10]. A light coherence-based nature of OCT provides great special resolution but very limited in opaque tissues imaging depth [11]. Efforts to overcome these fundamental limitations led to the investigation of virtual histology IVUS (VH-IVUS) and integrated backscatter IVUS (IB-IVUS), both of which involve analysis of the spectral content of backscattered ultrasound signals as a means of predicting tissue composition. Although VH-IVUS and IB-IVUS are clinically available methods intended to improve the ability of IVUS to detect plaque composition, the reliability of these approaches has recently been questioned $[12,13]$. Furthermore, intracoronary diffuse reflectance near-infrared spectroscopy (NIRS), combined with IVUS, has recently been clinically approved as a means of detecting lipid-rich plaques with the limitation being that the received signal, and therefore the detected lipid-distribution, is not depth-resolved [14].

Combined intravascular ultrasound and photoacoustic (IVUS/IVPA) imaging has been introduced as a hybrid imaging modality capable of addressing the ongoing clinical need for improved diagnostic imaging through its ability to supplement the morphological information provided by IVUS with the ability to assess lesion composition based on unique optical absorbance properties of endogenous plaque biomarkers or delivered contrast agents, which can be detected and localized using intravascular photoacoustic (IVPA) imaging [15, 16]. IVPA imaging relies on the absorption of energy from a nanosecond-pulsed laser, causing a rapid thermal expansion of surrounding tissue which results in the generation of an acoustic transient pulse that can be subsequently detected and localized by an IVUS transducer, used also for a pulse-echo ultrasound imaging. Combined IVUS/IVPA is capable of utilizing shared hardware, such as the transducer and ultrasound receiver, as well as shared digitization and image processing while providing spatially co-registered, 
complimentary information regarding the imaged vessel. To date, IVUS/IVPA imaging has been utilized for the localization of exogenous optical absorbers such as coronary stents [17, $18]$ or systemically delivered contrast agents $[19,20]$. The technique has been most widely investigated for the localization of lipid distribution within atherosclerotic plaques based on the unique optical absorbance peaks of lipid at approximately $1210 \mathrm{~nm}$ or $1720 \mathrm{~nm}$ in ex vivo [21-26] or in vivo studies [23].

While several potential applications of IVUS/IVPA imaging have been introduced, the continued development of the technique and, particularly, its translation to clinical practice necessitates the development of a system and integrated catheter suitable for real-time imaging. While several prototype integrated IVUS/IVPA catheter designs have been reported [21, 24, 27, 28], none of these designs have been suitable for the rapid rotation which would be required for real-time imaging (i.e. $\geq 30 \mathrm{~Hz}$ ), nor have they been coupled to systems capable generating both IVUS and IVPA signals or processing the data in real time at such speeds. At the same time, the real-time option for combined IVUS/IVPA imaging is a must for clinical transfer of the technology due to substantial decrease of procedure time and, therefore, risk for a patient, and ability to perform the IVPA imaging over QRS cardio cycles without imaging artefacts. Most demonstrations of IVUS/IVPA imaging have utilized experimental systems with Nd:YAG pumped optical parametric oscillators (OPO) tunable laser systems which operated at pulse repetition rates of $10-20 \mathrm{~Hz}[15-25,29,30]$. Recently, a $2 \mathrm{kHz}$ optical source based on a Raman laser pumped by a fast diode-pumped solid state (DPSS) Nd:YAG laser was introduced for IVPA imaging, enabling IVUS/IVPA imaging capable of a cross-sectional frame acquisition of approximately one second [31, 32]. However, assuming 256 IVPA A-lines per cross-sectional frame and no averaging, as is acquired by most clinical IVUS imaging systems, an optical source operating at nearly 8 $\mathrm{kHz}$ is required in order to enable real-time IVPA imaging. These A-lines are required to be acquired, processed and displayed as IVUS and IVPA images in Cartesian coordinates in real time. Herein, we present a first-in-kind IVUS/IVPA imaging system and integrated IVUS/IVPA imaging catheter capable of real-time ( $230 \mathrm{~Hz}$ ) imaging with online image processing and display of IVUS, IVPA and/or IVUS/IVPA images.

\section{Materials and Methods}

\section{A. Real-Time IVUS/IVPA Imaging System Design}

The overall system consists of a $10 \mathrm{kHz}$ pulsed laser for IVPA signal generation, an ultrasound pulser/receiver for transmitting IVUS pulses as well as receiving IVUS and IVPA signals, a spindle comprising a servo motor, encoder, optical laser-to-catheter coupler, and a radio frequency $(\mathrm{RF})$ rotary joint for data transfer to a custom designed controller platform capable of independent IVUS and IVPA signal synchronization, digitization, and image processing in real-time.

Laser Source-The system utilizes a $1064 \mathrm{~nm}$ DPSS laser with an 8 nanosecond pulse duration, controllable pulse repetition frequency from single-shot to beyond $10 \mathrm{kHz}$, with output energy greater than $2 \mathrm{~mJ}$ per pulse over the entire PRF range (SOL40W-1064, RPMC Lasers, Inc.). 
Spindle-A diagram of a custom designed spindle is shown in Fig. 1. The spindle was built for catheter torque cable rotation, optical and electrical coupling at the proximal end of the rotating catheter, and synchronization of excitation (i.e., ultrasound transmit, laser pulse) to angular position. The spindle was fabricated in house, and consisted of a frameless, brushless three-phase servo motor (Inland RBE-1812, Kollmorgen, Inc.), angular contact bearings (2208hdm, Barden, Inc.), a hollow-bore 8192 line-count angular encoder (model HS25, BEI sensors, Inc.), and a hollow RF rotary joint (custom part MT007U-

S12-1800RPM, Moflon / Jinpat Electronics, Inc.). The spindle bore is hollow end-to-end, permitting optical coupling via stationary air-beam focusing onto the rotating fiber core. The servo loop is closed threefold: hall sensor feedback for initial commutation angle, angular encoder for sinusoidal three-phase commutation and position/velocity feedback.

Optical handling of the spindle is $17+\mathrm{W}$, thus PA illumination is limited by the performance of the light delivery system inside the integrated imaging catheters. The electrical performance is limited by the RF rotary joint, which was designed to pass RF signals with a maximum frequency of $40 \mathrm{MHz}$. The overall reliability of the spindle was tested using a phantom with four wires located at cardinal directions and a phantom with stent with known struts geometry.

System Synchronization-Synchronization between spindle angle, RF digitizer, ultrasound pulser/receiver (5073PR, Panametrics-NDT, Inc.), pulsed laser, and storage subsystems is performed in hardware, removing any need for trace to trace re-alignment, etc. The core of the synchronization subsystem is an $80 \mathrm{MHz}$ counter/timer card with 8 independent timing engines (NI PXI-6602, National Instruments, Inc.). From the master time base, the counter/timer card generates all further requisite system clocks and triggers. Diagrammatic representation of the overall timing control sequence is depicted in Figure 2. The timing engine takes in the quadrature angular encoder signal, which yields the instantaneous rotor position. The frame trace-density values can be controlled by the system operator and are used to fire the laser and ultrasound pulser at their individual correct angles. The timing engine also accepts return synchronization signals from the laser and pulser, indicative of actual laser/pulser firing time. The final function of the timing engine is to properly align the A-line digitization trigger for both modalities, and trigger the digitizer appropriately. Because the frame trace densities can be controlled independently, the ratio of IVUS to IVPA traces per frame can be readily adjusted via the system user interface.

RF Signal Acquisition and Image Rendering-RF traces were digitized at $200 \mathrm{MSa} / \mathrm{s}$ and 12-bit depth via a high-speed digitizer with $2 \mathrm{MB}$ on-board memory per channel (NI PXI-5124, National Instruments, Inc.). A single channel was used for both ultrasound (US) and photoacoustic (PA) trace acquisition. However, unlike the system described in our previous publications [15-19, 23, 24, 29, 33], each signal was acquired in separate traces. This permitted the frame's A-line count to be different for the two modalities, while simultaneously minimizing data transfer across the PXI bus to the system controller RAM (NI PXIe-8135, National Instruments, Inc.). The US and PA traces are continuously transferred across the bus, thus avoiding limitations associated with the $2 \mathrm{MB}$ on-board memory of the digitizer. As a result, the system's maximum imaging rate - a product of 
frame-rate, trace-density, and imaging depth - is ultimately the bus bandwidth between the digitizer and controller.

The synchronization subsystem was designed such that each imaging modality had its respective A-lines equally spaced angularly, even if the A-line count is different for each modality. This indicates that the digitizer could be triggered at an irregular rate if the number of PA and US traces are not the same.

Following digitization of the IVUS and IVPA signals, RF signal processing, envelope detection, polar-to-Cartesian conversion, logarithmic compression, and display rendering are performed to enable live display as well as recording of uncompressed raw RF trace data for further processing. All steps after digitization are performed in software. Accomplishing all steps in software required substantial algorithm optimization and optimal utilization of all CPU cores via a multi-threaded pipeline. The pipeline's first stage transfers traces from the digitizer and collates them into frames of their respective modalities. These are then bandpass filtered in the Fourier domain using matched filtering to optimize SNR. The polar frame data is alias protected using an anisotropic kernel, implemented in the Fourier domain. Computationally efficient envelope detection is facilitated by enforcing analyticity, thus no Hilbert transform is needed per se. In addition to the standard B-mode real-time image display, the system also provides longitudinal Z-R views via catheter pullback, which can be done any number of times without stopping the system. Alternatively, this functions as Mmode imaging if the catheter isn't being retracted. Although frame trace-density and imaging depth cannot be changed while the system is running, many other settings are dynamically controllable: window level, window range, frame rate, receiver filter aperture and shape, noise removal, and display persistence.

Image Processing - The largest noise source is burst noise, coupled inductively when the PWM brushless motor's coil excitations change between discrete levels. Such noise is picked-up into the system on the catheter, prior to receiver amplification. After envelope detection, it manifests solely as a positive addition to the desired signal. In many respects it is very similar to salt noise, though arising from a different cause. The two noise mitigation options are, therefore, effectively salt filters that operate in time (frame-to-frame) and/or angle (trace-to-trace, within a single frame), each of which helps remove this in-band uncorrelated noise. The strength of these filters is set to the desired level by the user during imaging, as needed. These two filters are combined with a persistence operation, effectively weighing newer frames over older frames during the 'salt' removal process. A lower persistence setting will permit faster motions to be clearly visible, while simultaneously reducing the effectiveness of the 'salt' noise removal process.

\section{B. Integrated Catheter for In Vivo Real-Time Clinical IVUS/IVPA Imaging}

The integrated IVUS/IVPA imaging catheter utilized a design in which the optical fiber and coaxial cable were contained within a $\sim 1 \mathrm{~mm}$ outer diameter torque cable suitable for the rapid and continuous rotation rates required for real-time intravascular imaging (Fig. 3).

A one-meter-long one-directional dual-layer torque cable (type 255, Asahi-Intecc, Inc.) was used to transmit the torque from the spindle assembly to the distal end of the catheter. The 
drive cable had outer/inner diameter of $1.06 / 0.59 \mathrm{~mm}$. At the distal end, a $5 \mathrm{~mm}$ long, $1 \mathrm{~mm}$ outer diameter custom-made, hollow fixture was welded to the torque cable and held the ultrasound transducer and distal end of optical fiber. One side of the fixture contained a 3.5 $\mathrm{mm}$ long opening, serving as an optical and ultrasound window. On the proximal end of the torque cable, a $1 \mathrm{~mm}$ outer diameter, $30 \mathrm{~mm}$ long pipe served as a contact point to fix and rotate the catheter within the spindle. The direction of rotation was chosen such that the outer layer was compressed while inner layer expanded.

A diagram of the distal end of the integrated catheter is shown in Figure 3A. The optical fiber (FIP300330370, Molex, Inc.) was made out of fused silica with NA of 0.22 . The core and outer diameters of the fiber are 300 and 370 micrometers, respectively. Given the laser damage threshold of $0.5 \mathrm{GW} / \mathrm{cm}^{2}$, the optical fiber is capable of delivering up to $1.7 \mathrm{~mJ} /$ pulse. The distal tip of the fiber was polished at $37.5^{\circ}$ to optimize light delivery and minimize artefacts from internal reflections. To enable total internal reflection from the polished surface, a gas-trapping cap was installed to seal the distal tip of the optical fiber using epoxy. The cap was manufactured out of a glass pipe with outer and inner diameters of 0.6 and $0.4 \mathrm{~mm}$. respectively [24].

A single-element ultrasound transducer with center frequency of $40 \mathrm{MHz}$ and bandwidth of about $40 \%$ was used both to detect photoacoustic transients and for pulse/echo ultrasound imaging. This 0.5-mm-diameter transducer (repurposed from Atlantis ${ }^{\mathrm{TM} S R}$ Plus, Boston Scientific, Inc.) was connected to a coaxial cable with an outer diameter of approximately 180 micrometers and $50 \mathrm{Ohm}$ impedance.

On the proximal end, the optical fiber was polished flat, perpendicular to its longitudinal axis and fixed into an optical rotary joint. Finally, the fiber was coupled to a laser beam using a lens with a focal length of $75 \mathrm{~mm}$. The coaxial cable was connected to a pulser/ receiver (5073PR, Panametrics-NDT, Inc.) through the electrical slip ring rotary joint contained within the spindle. Photographs of the fully integrated catheter in Figure 3 show optical illumination out of the distal tip in close up (Fig 3B), as well as showing flexibility (Fig 3C).

Finally, since in vivo implementation of the combined IVUS/IVPA imaging requires a sheath to protect the artery from the high-speed rotating catheter, we tested the fully assembled integrated catheter with a first prototype of such sheath. The protecting sheath was made out of Polymethyl Pentene (PMP), trade mark TPX ${ }^{\mathrm{TM}}$, tubing (custom order, HottyPolymer, Inc.). The distal end of the catheter was introduced into an immobile $\mathrm{TPX}^{\mathrm{TM}}$ tubing with outer and inner diameters of 1.3 and $1.1 \mathrm{~mm}$, respectively. TPX ${ }^{\mathrm{TM}}$ was chosen as it is light transparent [34] and commercially used for IVUS imaging due to its low acoustic impedance.

In experiments, the integrated catheter was placed into a $\sim 5$-mm-diameter supporting polyethylene tubing where it was rotating unobstructed. The $\sim 20-\mathrm{cm}$-free distal end was introduced through another, $\sim 3-\mathrm{cm}$-long polyethylene tubing with inner diameter of $1.1 \mathrm{~mm}$ which directed the final $\sim 5 \mathrm{~cm}$-long bare naked distal end of the catheter into a water tank with phantoms. The protective TPX ${ }^{\mathrm{TM}}$ sheath covered only the distal end where it entered 
phantoms, such that both light and ultrasound propagating through the sheath during imaging.

\section{Imaging Performance Characterization}

System Calibration-Two separate phantoms were used to evaluate the system's imaging performance. The first phantom was built to provide verification of correct radial scaling of IVUS/IVPA images. It consisted of four $125-\mu \mathrm{m}$-diameter metal wires placed at various distances of $1.16 \mathrm{~mm}, 2.46 \mathrm{~mm}, 3.88 \mathrm{~mm}$, and $5.48 \mathrm{~mm}$ from the axis of rotation as ascertained from microscope images of the phantom itself. Each wire was parallel to the catheter and positioned at one of the cardinal directions. The second phantom was utilized to assess the point spread function (PSF) of the real-time IVUS/IVPA imaging system and catheter. It consisted of a single $10 \mu \mathrm{m}$ carbon fiber suspended parallel to and approximately $4.5 \mathrm{~mm}$ from the integrated IVUS/IVPA catheter. The system's PSF was assessed by imaging this carbon fiber target in water, and measured directly from the final screen display images, thus truly indicates live performance.

Vessel-Mimicking Phantom with Embedded Stent. A hollow cylindrical phantom was constructed to mimic the geometry of the vessel as well as the acoustic and optical scattering properties of arterial tissue using a previously described procedure [18]. Briefly, the vessel phantom was comprised of $8 \%$ polyvinyl alcohol with $0.1 \% 15 \mu \mathrm{m}$ silica particles, with an inner diameter of $5 \mathrm{~mm}$ and a wall thickness of approximately $3 \mathrm{~mm}$. Subsequently, a coronary stent (BX Velocity, Cordis) was expanded within the lumen of the vesselmimicking phantom.

\section{Results}

\section{A. Evaluation of System Performance}

The system controller was capable of simultaneously controlling all system hardware while performing image rendering and display in real-time. For dual-modality real-time imaging at 256 lines per frame, the digitizer acquires 15,360 RF traces are required per second. The system was tested and shown to achieve continuous acquisition above 25,000 RF traces per second, indicating that the current hardware capabilities will suffice foreseeable future needs. The imaging speed - in terms of frame-rate, trace-density, and imaging depth - of the system is currently limited by the PXI-based digitizer's maximum bandwidth of $132 \mathrm{MB} / \mathrm{s}$. The PXI bus bandwidth limitation effectively places a cap on the product of the three factors (frame-rate, trace-density, and imaging depth). Although the controller and chassis are PXI Express (PXIe), the digitizer is based on the older PXI standard and is thus responsible for the maximum imaging rate.

In contrast to our prior IVUS/IVPA system implementations[15-18, 23, 24, 29, 33], the present system enables efficient digitization of separate IVUS and IVPA traces, independent IVUS and IVPA trace densities. Relative to prior systems, there is a substantial advantage in acquiring IVUS and IVPA signals in separate digitizer trace acquisitions: eliminating wasted digitizer trace memory and bandwidth between the digitizer and the CPU which results from storing the dead-time portion between the two modalities (the time allotted for late IVUS 
echoes). Additionally, the two modalities need not have the same frame trace-densities. The cases shown in Figure 4 are all instances of integer ratios between the modalities - which generally must be true if homogeneous distribution of A-lines per frame is desired. This is useful as azimuthal resolution is typically worse for IVPA than IVUS [15] - thus our solution permits optimal IVPA and IVUS trace-densities while removing excess PA traces, thus lowering laser PRF requirements without sacrificing IVUS image quality. In cases where IVPA azimuthal resolution is superior, the system can be set to acquire more IVPA traces than IVUS.

Incorporation of the IVUS transducer coaxial cable and optical fiber into a $1 \mathrm{~mm}$ diameter torque cable provided the requisite rotational stability needed to enable real-time imaging while maintaining catheter flexibility and cross sectional profile necessary for future clinical applications of intravascular imaging. Both optical simulation studies and physical experiments were utilized to determine the optimal polishing angle for the distal end of the optical fiber, $37.5^{\circ}$, to minimize reflections and maximize light propagation into the path of the ultrasound beam.

\section{B. Evaluation of Imaging Characteristics}

All IVPA and IVUS/IVPA results shown are screenshots of the display, or exported images saved to disk in real-time. There is no off-line processing of any kind applied to the presented images. All image processing is performed in real-time and images presented below are in the identical form to how they appeared on the system display at the time of data acquisition.

The PSF was assessed by imaging the carbon fiber target with the system running at a 17.5 $\mathrm{Hz}$ framerate, $10 \mathrm{~mm}$ imaging diameter, dynamic range set to $30 \mathrm{~dB}$, salt removers disabled, and display persistence at $220 \mathrm{~ms}$. When measured at $6 \mathrm{~dB}$ below peak, $42 \mu \mathrm{m}$ radial and $3.1^{\circ}$ azimuthal resolution was achieved. Ascertained at $20 \mathrm{~dB}$ below peak, the PSF registered $120 \mu \mathrm{m}$ radial and $4.2^{\circ}$ azimuthal (Fig. 5). This framerate was chosen to avoid any ring artefacts that appear at higher framerates which are discussed below.

Representative screen captures of IVUS and IVPA images taken from the real-time system during imaging of the stented vessel-mimicking phantom at 24 frames per second and at 30 frames per second are shown in Fig. 6A and Fig. 6B and in 6D and 6E, respectively. Different cross-sections of the same phantom are shown. Both the IVUS and IVPA images are displayed using a $30 \mathrm{~dB}$ dynamic range with a $10 \mathrm{~mm}$ diameter field of view and a raster size of $512 \times 512$ pixels. While the lumen boundary of the phantom can be seen in the IVUS image, the metal stent struts are clearly demarcated by the corresponding IVPA image, and the combined IVUS/IVPA images enable assessment of the strut placement in the context of the phantom wall (Fig. 6C and 6F). The pulse energy from the distal end of the integrated catheter was $100 \mu \mathrm{J}$ per pulse. Overall, IVUS/IVPA imaging using the real-time system with rapid rotation of integrated catheter has yielded sufficient image quality compared with results previously obtained using slower data acquisition and off-line processing/rendering.

Clinical intravascular imaging systems must contain the distal end of the catheters within a stationary sheath to mitigate risk of injury to the vascular wall during translation and/or 
rotation of the catheter. In order to further evaluate the clinical viability of the integrated IVUS/IVPA catheter design, IVUS and IVPA images of the wire phantom were obtained with the catheter free (Fig. 7A) and contained within a TPX ${ }^{\mathrm{TM}}$ sheath (Fig 7B). These images are shown with dynamic range of $\sim 20 \mathrm{~dB}$. However, the window level of the image display was changed between acquisition of the images without and with the TPX ${ }^{\mathrm{TM}}$ sheath shown in Figure 7. The signal-to-noise ratio (SNR) of the images obtained with the catheter confined within TPX ${ }^{\mathrm{TM}}$ tubing was approximately $6 \mathrm{~dB}$ lower than that of the unobstructed catheter for both the IVUS images (left) and IVPA images (right).

\section{Efficiency of Noise Removal Algorithms}

Noise immunity is critical for ultrasound and photoacoustic imaging systems, and mitigation of system noise was specifically addressed in our system design and implementation through filtering and isolation of electrical components. Medical imaging systems must be robustly tolerant to radio frequency interference and other external noise sources. The small $1 \mathrm{~mm}$ catheter outer diameter necessitated micro-coax for electrical connection to the PZT transducer. Most problematic is in-band noise which enters the system before the amplifier (i.e., picked up on the catheter). The ultrasound receiver subsequently amplifies this in-band noise, which cannot be removed at the signal processing stage due to being in-band. Usually the best noise mitigation is that which prevents entry in the first place, rather than necessitate removal thereafter. However, catheters are disposable and must be relatively cheap, therefore substantial effort was put into incorporating robust, yet computationally cheap, noise removal algorithms operating in real-time. Figure 8 demonstrates an extreme example of a wire-target phantom without (A) and with (B) the salt filter algorithms applied in a particularly poor noise situation. The noise seen in Figure 8A is in-band burst noise, picked up before the receiver's preamplifier. The bulk of the in-band noise picked up by the system is generated from the three-phase pulse-width modulation (PWM)-based servo drive. The high-frequency content from the PWM edges is RF-coupled onto the catheter coax and picked up before the receiver's preamplifier. This noise is poorly correlated, and is easily removed by the on-line salt-remover software algorithms, with minimal degradation to image quality.

\section{Discussion}

The developed system comprises an integrated IVUS/IVPA imaging catheter, a $10 \mathrm{kHz}$ PRF laser source and receive electronics capable of real-time image processing, display and storage. At the distal end of the integrated catheter, both the ultrasound transducer with the coaxial cable and the optical fiber with gas-trapping cap were mounted to the torque cable fixture which enabled alignment of the optical and ultrasound beams while maintaining overall catheter flexibility (Fig. 3). Although optical transmission efficiency has proven relatively uniform azimuthally, the current integrated catheter design does complicate optical alignment during catheter installation or replacement. The overall system design enabled independent triggering and acquisition of IVUS and IVPA images (Fig. 2), thereby allowing variable trace densities per frame for each modality (Fig. 4). 
The real-time IVUS/IVPA imaging system and integrated catheter yielded a PSF similar to previous reports of IVPA imaging resolution. At $6 \mathrm{~dB}$ below the peak signal, imaging the 10 $\mu \mathrm{m}$ carbon fiber target with the present system and a catheter without TPX ${ }^{\mathrm{TM}_{\text {sheath }}}$ exhibited axial and azimuthal resolutions of $42 \mu \mathrm{m}$ and $3.1^{\circ}$, respectively. By comparison, an early report on IVPA imaging using an external light beam and an off-the-shelf IVUS transducer to image a similar target measured the $6 \mathrm{~dB}$ down resolution at $40 \mu \mathrm{m}$ and $3.1^{\circ}$ [13].

The images displayed in Figure 6 were achieved at 24 (A-C) and at 30 (D-F) frames per second. The ring artefact observed in the IVUS image at 24 frames per second and in both IVUS and IVPA images at 30 frames per second is due to inductive coupling of the encoder signal into the catheter's coax. At $24 \mathrm{~Hz}$ framerate, an 8192 line-count two-channel encoder will have edge-time spacing of $5.09 \mu \mathrm{s}$. This corresponds to rings spaced at $3.81 \mathrm{~mm}$ intervals for IVUS and $7.63 \mathrm{~mm}$ intervals for IVPA. The artefact is seen as expected in the IVUS image, however it is out of range for IVPA ( $5 \mathrm{~mm}$ radial depth-of-view). At a $30 \mathrm{~Hz}$ framerate, the encoder edge-time ring artefacts appear at intervals of $3.05 \mathrm{~mm}$ and $6.10 \mathrm{~mm}$, for IVUS and IVPA, respectively, and hence obstructs view of large-lumen arteries/ phantoms. This problem is easily solved - replacing the unnecessarily high-resolution encoder with a low-resolution, 2048 line-count model would result in worst-case artefact ring spacing of $12 \mathrm{~mm}$ for IVUS. Thus, the artefact would not be encountered for imaging diameters below $24 \mathrm{~mm}$.

The catheter's protective sheath will both obstruct light propagation and attenuate ultrasound waves. Thus, the optical attenuation in TPX ${ }^{\mathrm{TM}}$ sheath at $1064 \mathrm{~nm}$ is modest. Indeed, according to [34], TPX ${ }^{\mathrm{TM}}$ exhibits the transparency of about $90 \%$ in the visible to NIR region. To confirm that, we tested the transparency of TPX ${ }^{\mathrm{TM}}$ and found losses to be about $0.86 \mathrm{~dB}$ and $0.51 \mathrm{~dB}$ at $1720 \mathrm{~nm}$ in a dry (both catheter and TPX ${ }^{\mathrm{TM}}$ tubing were out of water) and wet (catheter with TPX ${ }^{\mathrm{TM}}$ tubing were in water and water was injected between tubing and catheter) environment, respectively. While optical attenuation in ТРX ${ }^{\mathrm{TM}}$ tubing was not significant, the presence of the tubing can result in altered light distribution due to scattering and refraction which was notable in water. This was confirmed visually - the TPX ${ }^{\mathrm{TM}}$ tubing appears a bit blurry. Both axial and lateral resolution of IVUS imaging in the presence of the $\mathrm{TPX}^{\mathrm{TM}}$ sheath are $30 \%$ to $50 \%$ worse compared to that without the sheath. At the same time, light scattering in blood is expected to be strong and, therefore, will dominate absorption in optical attenuation [35]. Nevertheless, the reported $6 \mathrm{~dB}$ SNR decrease in the presence of TPX ${ }^{\mathrm{TM}}$ tubing is critical, and the material for the protecting sheath as well as the design of the whole unit and manufacturing technology of the tubing are still a subject to investigate.

While IVUS imaging is currently recognized as a clinically approved procedure, the safety of IVPA imaging has not been confirmed yet. Moreover, compared to IVPA imaging results previously published by different groups [17, 22, 25, 28, 36] using slow laser PRF's and excised tissues, real-time in vivo IVPA imaging requires around 5000 pulses per second. Therefore, it is a must to confirm that such radiation will not result in tissue damage.

During real-time imaging, the catheter rapidly rotates around its longitudinal axis and is simultaneously pulled back thus does not expose one single area of soft tissues for an 
extended period. The energy will be distributed along a relatively large area. For the current design of our integrated imaging catheters, the beam size measured in air $2 \mathrm{~mm}$ away from the catheter is found to be $1.5 \mathrm{~mm}$ by $2 \mathrm{~mm}$ (axial and azimuthal, respectively). The pullback speed was $0.5 \mathrm{~mm} / \mathrm{s}$ - a standard speed for IVUS. Also, while this laser is capable of working at a PRF of up to $10 \mathrm{kHz}$, the PRF required for real-time imaging depends on the number of A-lines per image and the framerate. For a $30 \mathrm{~Hz}$ framerate and $128 \mathrm{~A}$-lines per image, one single point of soft tissues will be exposed by 60 trains of light pulses evenly distributed within 2 seconds wherein each train consists of 15 pulses arriving at $3840 \mathrm{~Hz}$. In this estimation, it was assumed that the pulses were evenly distributed in time. According to ANSI standards [37], maximum permitted exposure (MPE) for our case was calculated to be $18 \mathrm{~mJ} / \mathrm{cm}^{2}$ at $1064 \mathrm{~nm}$ while in our experiments the fluence was only $3.3 \mathrm{~mJ} / \mathrm{cm}^{2}$.

Therefore, IVPA imaging is possible well below the MPE as given by ANSI standards.

However, safety is a very important issue and our future work is to carefully investigate it using excised tissues and histological analysis. Another future direction will focus on characterization of the overall system performance for monitoring stent deployment as well as its utilization to localize intra-plaque macrophages labeled with exogenous contrast agents designed to provide high IVPA contrast $[19,20,30]$. We will also be integrating a modified laser source operating at an application-specific optical wavelength (e.g., $1210 \mathrm{~nm}$ or $1720 \mathrm{~nm}$ for imaging lipid) to further enable improved vessel characterization.

\section{Conclusions}

Combined IVUS/IVPA imaging has been previously demonstrated in preclinical experiments as a hybrid modality which enables complimentary characterization of arterial anatomy and composition. However, previously reported IVUS/IVPA imaging systems have been limited by the use of low rep-rate optical sources and either stationary or slowly rotated integrated catheters. This has resulted in overall IVUS/IVPA imaging frame rates on the order of several seconds, a factor compounded by off-line image processing.

Together, the IVUS/IVPA imaging system and integrated catheter described herein are capable of rapid and continuous rotation, data acquisition, and image rendering at frame rates of $30 \mathrm{~Hz}$ or greater. As the first demonstration of real-time IVUS/IVPA imaging, these results represent a significant step towards its development into a clinically-viable modalityenabling the rapid collection of data for in vivo validation studies and a more direct comparison with existing clinical intravascular imaging techniques.

\section{Acknowledgments}

This work was supported in part by the National Institutes of Health (NHLBI) under grant R01HL124417.

\section{References}

1. Roger VL, Go AS, Lloyd-Jones DM, Benjamin EJ, Berry JD, Borden WB, et al. Heart Disease and Stroke Statistics-2012 Update: A Report From the American Heart Association. Circulation. 2012; 125:e2-e220. [PubMed: 22179539]

2. Kolodgie FD, Virmani R, Burke AP, Farb A, Weber DK, Kutys R, et al. Pathologic assessment of the vulnerable human coronary plaque. Heart. 2004; 90:1385-1391. [PubMed: 15547008] 
3. Ross R. Cell Biology of Atherosclerosis. Annual Review of Physiology. 1995; 57:791-804.

4. Mullenix PS, Andersen CA, Starnes BW. Atherosclerosis as Inflammation. Annals of vascular surgery. 2005; 19:130-138. [PubMed: 15714382]

5. Packard RRS, Libby P. Inflammation in Atherosclerosis: From Vascular Biology to Biomarker Discovery and Risk Prediction. Clinical Chemistry. 2008; 54:24-38. [PubMed: 18160725]

6. Choudhury RP, Fuster V, Fayad ZA. Molecular, cellular and functional imaging of atherothrombosis. Nat Rev Drug Discov. 2004; 3:913-925. [PubMed: 15520814]

7. Waxman S, Ishibashi F, Muller JE. Detection and Treatment of Vulnerable Plaques and Vulnerable Patients: Novel Approaches to Prevention of Coronary Events. Circulation. 2006; 114:2390-2411. [PubMed: 17130356]

8. Glagov S, Weisenberg E, Zarins CK, Stankunavicius R, Kolettis GJ. Compensatory Enlargement of Human Atherosclerotic Coronary Arteries. New England Journal of Medicine. 1987; 316:13711375. [PubMed: 3574413]

9. Hackett D, Davies G, Maseri A. Pre-existing coronary stenoses in patients with first myocardial infarction are not necessarily severe. European Heart Journal. 1988; 9:1317-1323. [PubMed: 3229426]

10. Prati F, Arbustini E, Labellarte A, Dal Bello B, Sommariva L, Mallus MT, et al. Correlation between high frequency intravascular ultrasound and histomorphology in human coronary arteries. Heart. 2001; 85:567-570. [PubMed: 11303012]

11. Vancraeynest D, Pasquet A, Roelants V, Gerber BL, Vanoverschelde J-1J. Imaging the valneraable plaque. Journal of the American College of Cardiology. 2011; 57:1961-1979. [PubMed: 21565634]

12. Kim SW, Mintz GS, Hong YJ, Pakala R, Park KS, Pichard AD, et al. The virtual histology intravascular ultrasound appearance of newly placed drug-eluting stents. Am J Cardiol. 2008; 102:1182-6. [PubMed: 18940288]

13. Thim T, Hagensen MK, Wallace-Bradley D, Granada JF, Kaluza GL, Drouet L, et al. Unreliable assessment of necrotic core by virtual histology intravascular ultrasound in porcine coronary artery disease. Circ Cardiovasc Imaging. 2010; 3:384-91. [PubMed: 20460496]

14. Brugaletta S, Garcia-Garcia HM, Serruys PW, de Boer S, Ligthart J, Gomez-Lara J, et al. NIRS and IVUS for Characterization of Atherosclerosis in Patients Undergoing Coronary Angiography. JACC: Cardiovascular Imaging. 2011; 4:647-655. [PubMed: 21679900]

15. Sethuraman S, Aglyamov SR, Amirian JH, Smalling RW, SY E. Intravascular photoacoustic imaging using an IVUS imaging catheter. IEEE Trans Ultrason Ferroelectr Freq Control. 2007; 54:978-986. [PubMed: 17523562]

16. Sethuraman S, Amirian JH, Litovsky SH, Smalling RW, Emelianov SY. Ex vivo Characterization of Atherosclerosis using Intravascular Photoacoustic Imaging. Opt Express. 2007; 15:1665716666. [PubMed: 19550952]

17. Karpiouk AB, Wang B, Amirian J, Smalling RW, Emelianov SY. Feasibility of in vivo intravascular photoacoustic imaging using integrated ultrasound and photoacoustic imaging catheter. J Biomed Opt. 2012; 17:96008-1. [PubMed: 23085909]

18. Su JL-S, Wang B, Emelianov SY. Photoacoustic imaging of coronary artery stents. Optics Express. 2009; 17:19894-19901. [PubMed: 19997212]

19. Wang B, Yantsen E, Larson T, Karpiouk AB, Sethuraman S, Su JL-S, et al. Plasmonic intravascular photoacoustic imaging for detection of macrophages in atherosclerotic plaques. Nano Lett. 2009; 9:2212-7. [PubMed: 18844426]

20. Yeager D, Karpiouk AB, Wang B, Amirian JH, Sokolov K, Smalling RW, et al. Intravascular photoacoustic imaging of exogenously labeled atherosclerotic plaque through luminal blood. Journal of Biomedical Optics. 2012; 17:106016-106016. [PubMed: 23224013]

21. Jansen K, van der Steen AFW, van Beusekom HMM, Oosterhuis JW, van Soest G. Intravascular photoacoustic imaging of human coronary atherosclerosis. Opt Lett. 2011; 36:597-599. [PubMed: 21368919]

22. Jansen K, Wu M, van der Steen AFW, van Soest G. Lipid detection in atherosclerotic human coronaries by spectroscopic intravascular photoacoustic imaging. Opt Express. 2013; 21:2147284. [PubMed: 24104022] 
23. Wang B, Karpiouk AB, Yeager D, Amirian JH, Litovsky SH, Smalling RW, et al. In vivo Intravascular Ultrasound-guided Photoacoustic Imaging of Lipid in Plaques Using an Animal Model of Atherosclerosis. Ultrasound in medicine \& biology. 2012; 38:2098-2103. [PubMed: 23069134]

24. Karpiouk AB, Wang B, Emelianov SY. Development of a catheter for combined intravascular ultrasound and photoacoustic imaging. Rev Sci Instrum. 2010; 81:014901. [PubMed: 20113121]

25. Zhang J, Yang S, Ji X, Zhou Q, Xing D. Characterization of lipid-rich aortic plaques by intravascular photoacoustic tomography: ex vivo and in vivo validation in a rabbit atherosclerosis model with histologic correlation. J Am Coll Cardiol. 2014; 64:385-90. [PubMed: 25060374]

26. Jansen K, Wu M, van der Steen AFW, van Soest G. Photoacoustic imaging of human coronary atherosclerosis in two spectral bands. Photoacoustics. 2014; 2:12-20. [PubMed: 25302152]

27. Hsieh BY, Chen SL, Ling T, Guo LJ, Li PC. All-optical scanhead for ultrasound and photoacoustic dual-modality imaging. Opt Express. 2012; 20:1588-96. [PubMed: 22274501]

28. Wei W, Li X, Zhou Q, Shung KK, Chen Z. Integrated ultrasound and photoacoustic probe for coregistered intravascular imaging. J Biomed Opt. 2011; 16:106001. [PubMed: 22029348]

29. Wang B, Su JL-S, Amirian JH, Litovsky SH, Smalling RW, Emelianov SY. Detection of lipid in atherosclerotic vessels using ultrasound-guided spectroscopic intravascular photoacoustic imaging. Opt Express. 2010; 18:4889-4897. [PubMed: 20389501]

30. Yeager D, Chen Y-S, Litovsky SH, Emelianov SY. Intravascular photoacoustics for image-guidance and temperature monitoring during plasmonic photothermal therapy of atherosclerotic plaques: a feasibility study. Theranostics. 2013; 4:36-46. [PubMed: 24396514]

31. Piao Z, Ma T, Li J, Wiedmann M, Huang S, Yu M, et al. High speed intravascular photoacoustic inaging with fast optical parametric oscillator laser at $1.7 \mathrm{um}$. Applied Physics Letters. 2015; 107:083701, 1-4. [PubMed: 26339072]

32. Wang P, Ma T, Slipchenko MN, Liang S, Hui J, Shung KK, et al. High-speed Intravascular Photoacoustic Imaging of Lipid-laden Atherosclerotic Plaque Enabled by a 2-kHz Barium Nitrite Raman Laser. Sci Rep. 2014; 4

33. Wang B, Karpiouk AB, Yeager D, Amirian JH, Litovsky SH, Smalling RW, et al. Intravascular photoacoustic imaging of lipid in atherosclerotic plaques in the presence of luminal blood. Opt Lett. 2012; 37:1244-6. [PubMed: 22466209]

34. I. Mitsui Chemicals America. TPX ${ }^{\mathrm{TM}}$ : a functional resin that creates high-value-added products. 2011

35. Roggan A, Friebel M, Dorschel K, Hahu A, Muller G. Optical properties of circulating human blood in the wavelength range 400-2500 nm. Journal of Biomedical Optics. 1999; 4:36-46. [PubMed: 23015168]

36. Bai X, Gong X, Hau W, Lin R, Zheng J, Liu C, et al. Intravascular optical-resolution photoacoustic tomography with a $1.1 \mathrm{~mm}$ diameter catheter. Public Library of Science. 2014; 9:e92463, 1-6.

37. Americal National Standard for Safe Use of Lasers. Vol. Z136.1. Laser Institute of America ed; 2007. 


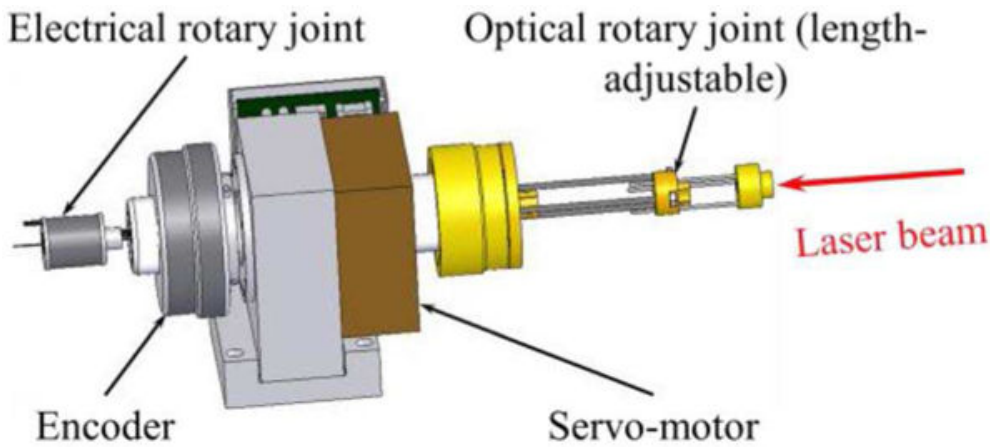

Fig. 1.

Diagram of the optical-electrical spindle. 


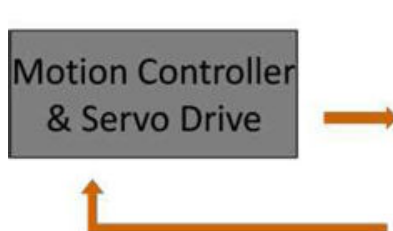

Motor
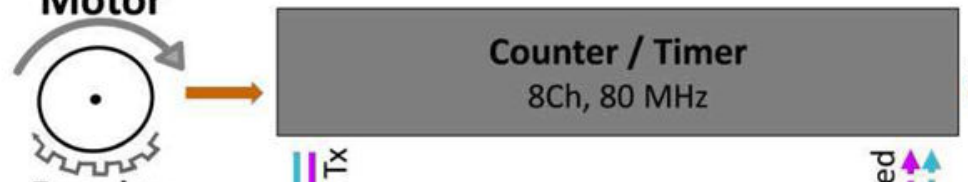

Encoder

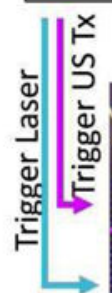

Tou Trigger

12-bit

Feedback

Fig. 2.

Diagram of the real-time IVUS/IVPA imaging system timing engine trigger signals: timing engine receives encoder signal, correctly triggers modality sources at requisite times, and coordinates proper digitizer trace acquisition synchronization. 

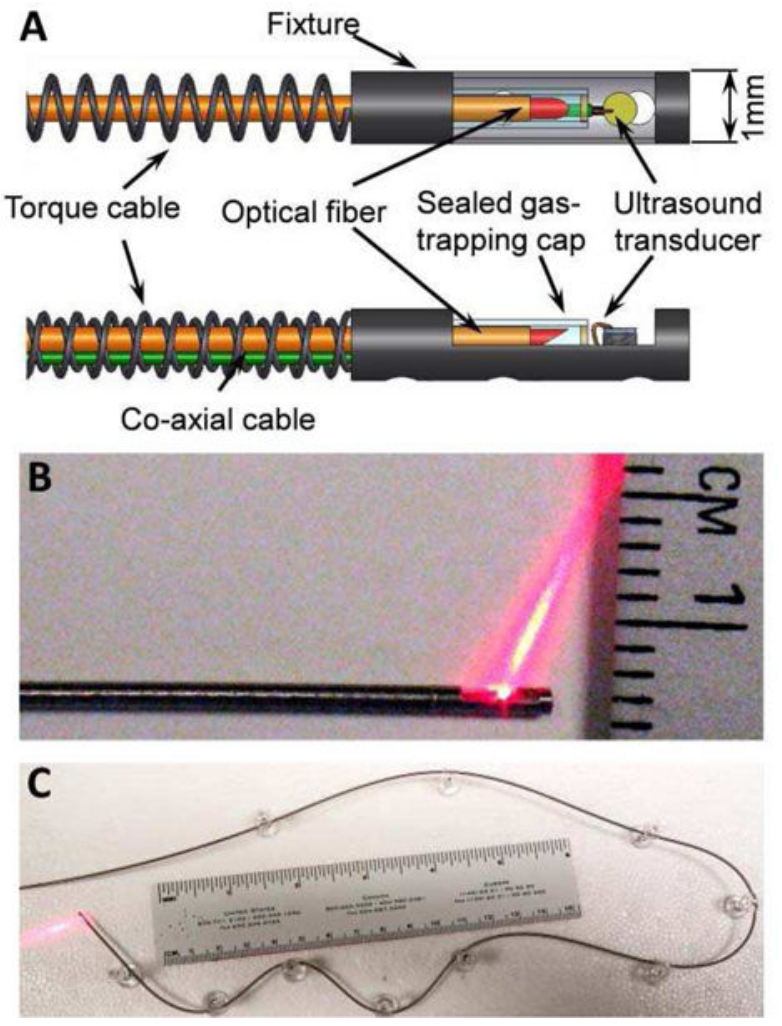

Fig. 3.

Integrated IVUS/IVPA catheter. (A) Schematic representation of the distal end design.

Photographs of (B) the distal end with optical illumination and (C) photograph showing the fully integrated IVUS/IVPA catheter flexibility. 

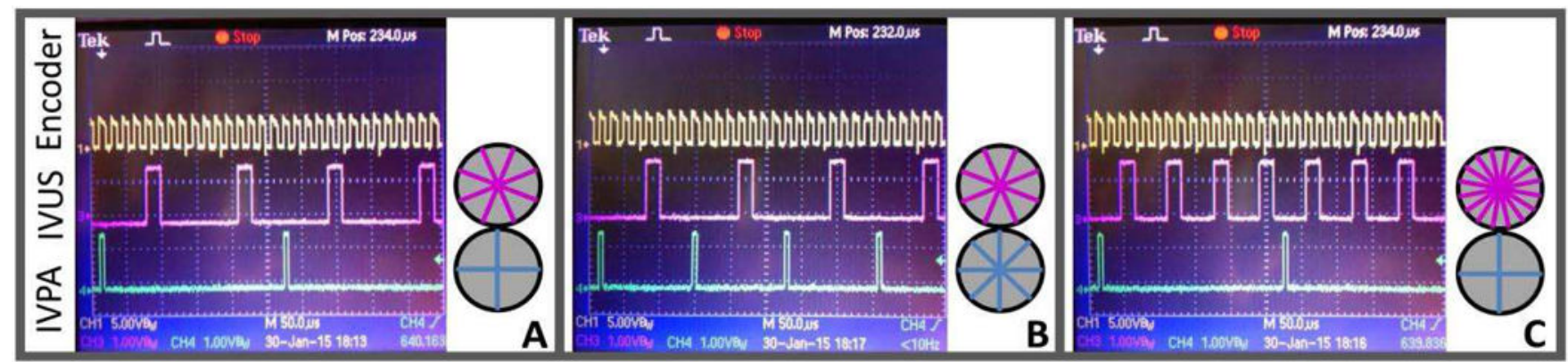

Fig. 4.

Depiction of actual trigger signals for $30 \mathrm{~Hz}$ framerate imaging in cases of trace-densities: (A) 256 US, 128 PA, (B) 256 US, 256 PA, (C) 512 US, 128 PA Differing trigger pulse-width requirements of the laser and ultrasound are correctly produced by the timing engine. Trace distribution diagrams are for illustration of relative ratio between modality A-line counts only. 
A

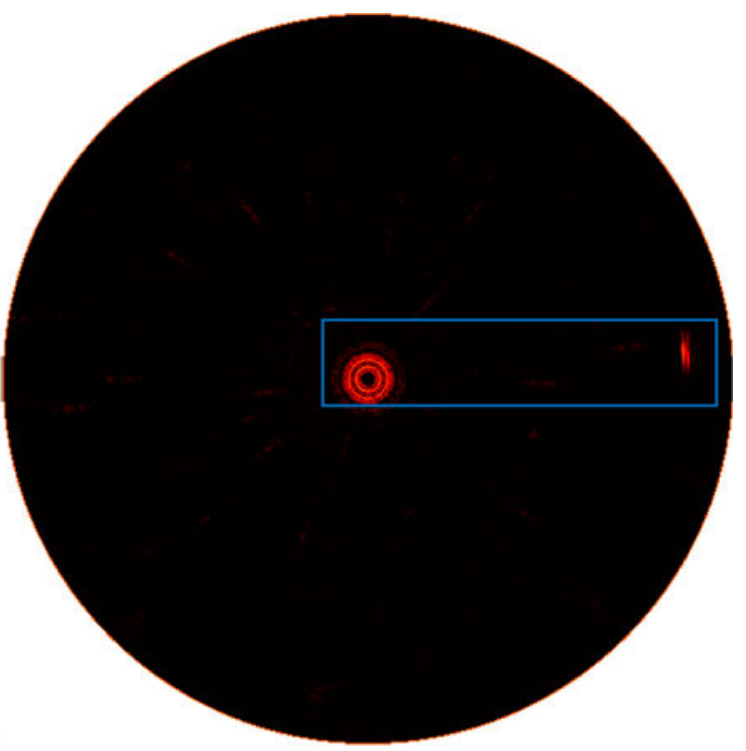

B

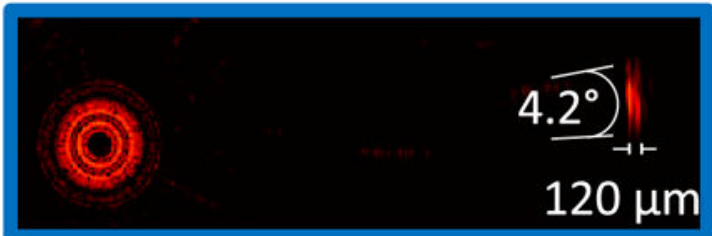

Fig. 5.

Assessment of PSF for IVPA imaging. (A) IVPA image of carbon fiber test target obtained in water, imaging at $17.5 \mathrm{~Hz}$ framerate with a $10 \mathrm{~mm}$ field of view. (B) Zoomed in view of the carbon fiber target, with indicated angular and axial resolutions measured at $20 \mathrm{~dB}$ below peak signal. 


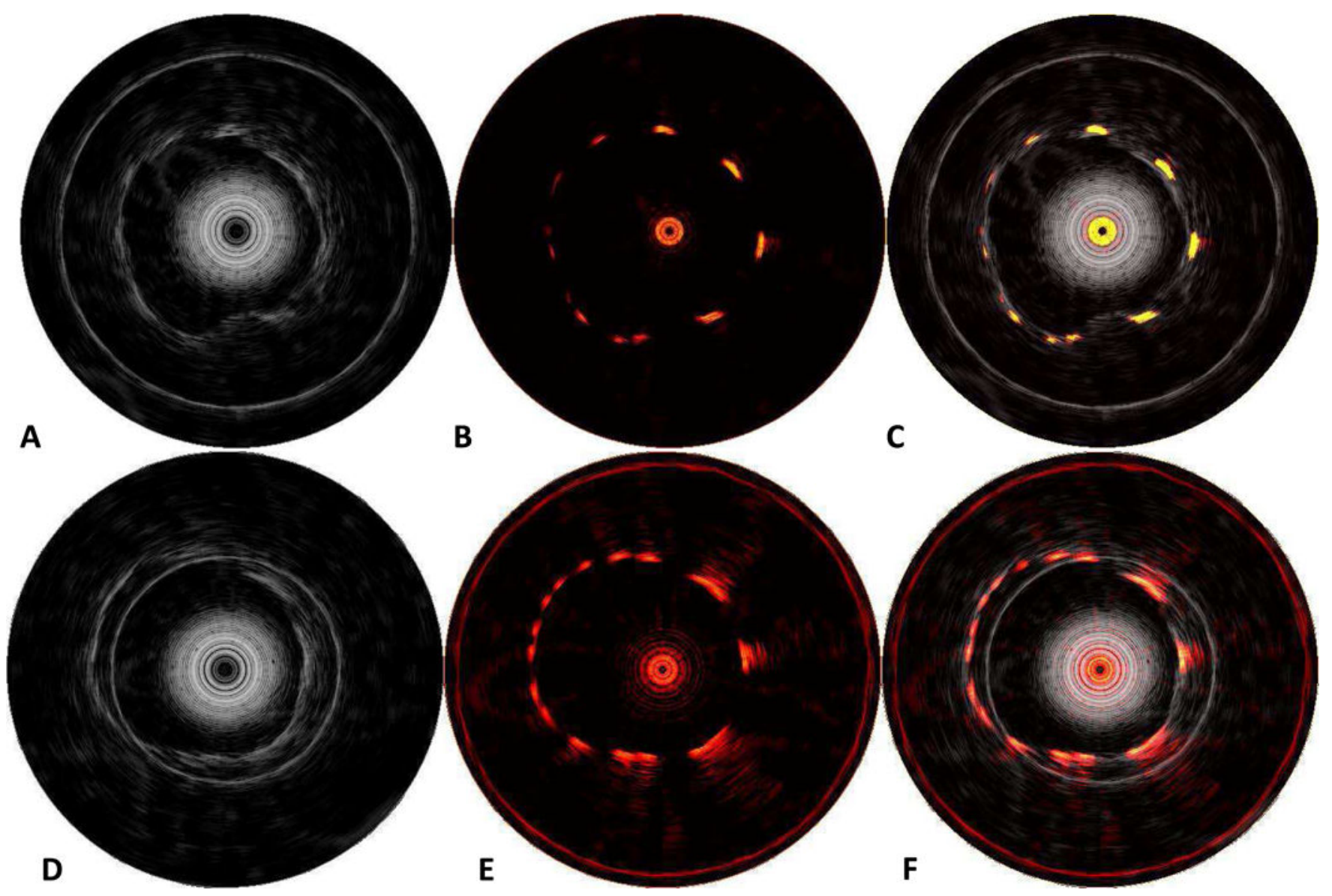

Fig. 6.

Results of imaging stent phantom at $24 \mathrm{~Hz}(\mathrm{~A}-\mathrm{C})$ and $30 \mathrm{~Hz}(\mathrm{D}-\mathrm{F})$ framerate. (A and D) IVUS image revealing phantom wall, (B and E) IVPA image revealing stent struts, and (C and F) combined IVUS/IVPA revealing stent strut apposition with the phantom wall. Dynamic range is $30 \mathrm{~dB}$ for all images. 

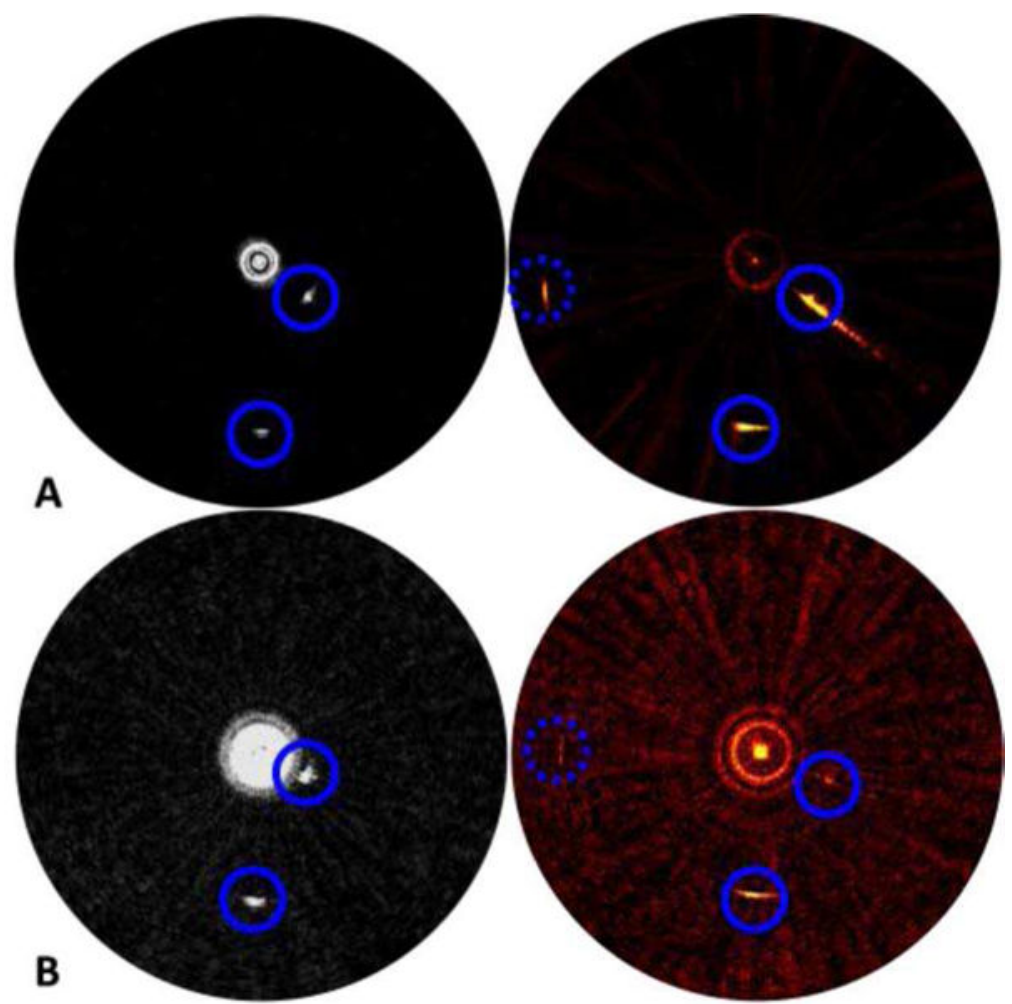

Fig. 7.

Performance degradation of catheter when operated inside TPX ${ }^{\mathrm{TM}}$ sheath. IVUS (left) and IVPA (right) images of a wire phantom obtained without (A) and with (B) a TPX ${ }^{\mathrm{TM}}$ sheath. Blue circles indicate the locations of the wire targets. All images are shown in dynamic scale of $\sim 20 \mathrm{~dB}$. 

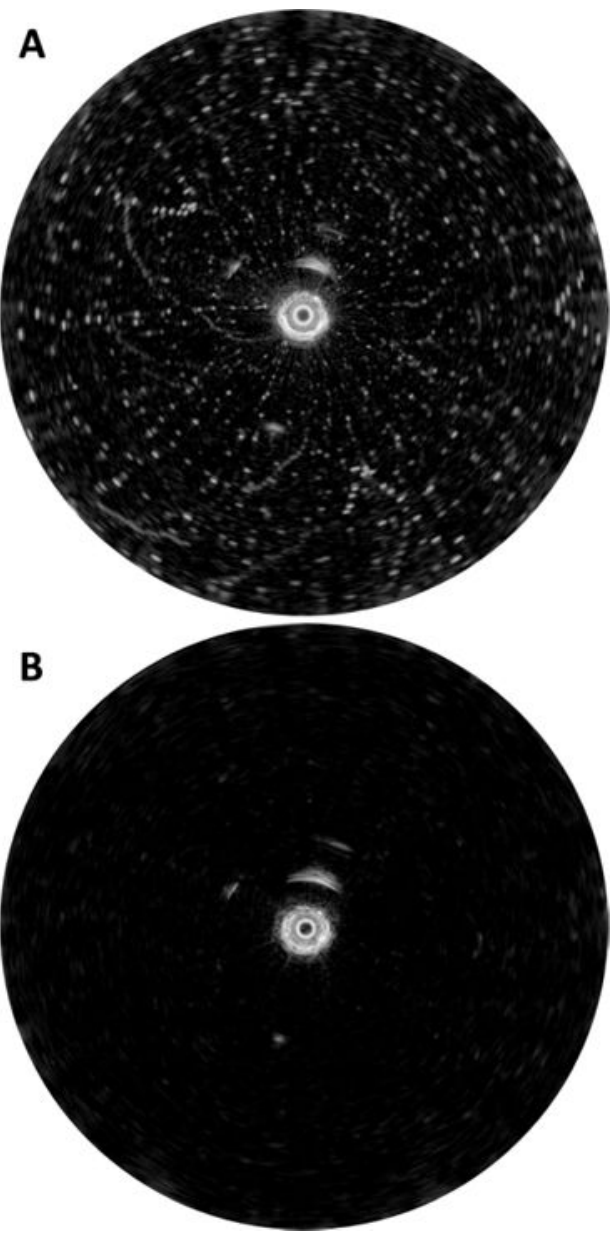

Fig. 8.

Example of particularly poor noise situation (A) without and (B) with the salt remover algorithms enabled. 